\title{
LE MANUSCRIT AUTOGRAPHE DU CORNET À DÉS, UNE COMMANDE DE PAUL BONET
}

Françoise NICOL*

"Le poète-enfant demeure un renaissant matin." Max Jacob, Derniers poèmes en vers et en prose

Tusqu'à sa mort, Max Jacob n'a cessé d'affirmer l'importance du Cornet à dés. Le manuscrit exceptionnel qui se trouve depuis 1990 au fonds Max Jacob de la Médiathèque d'Orléans ${ }^{1}$ l'atteste à tous égards. Réalisé probablement dans les derniers mois de la vie du poète, en 1943, il porte un titre remarquable : Le Cornet à dés par Max Jacob, 1898-1917. 1898 rappelle la rédaction précoce des premiers poèmes en prose, en tout cas le début de l'activité d'écriture parisienne de Max Jacob (il a vingt-deux ans) ; 1917, celle de l'édition originale. 1898-1943 : la présence du recueil de poèmes en prose dans la vie de son auteur est ainsi attestée pendant quarante-cinq ans.

\footnotetext{
Françoise Nicol est maître de conférences HDR à l'Université de Nantes et fait partie de l'équipe de recherches de l'Université de Rennes 2, le CELLAM. Après sa thèse sur les livres du peintre Georges Braque (2000), elle a travaillé sur les œuvres de poètes du Xx $x^{\mathrm{e}}$ siècle, principalement Pierre Reverdy et les surréalistes. En 2014, elle a publié son habilitation, Georges Limbour, l'aventure critique (PUR), à partir de l'édition, aux côtés de Martine Colin Picon des écrits sur l'art de Georges Limbour (Spectateur des arts, Le Bruit du temps, 2013). Sa recherche actuelle porte sur les arts visuels aux $\mathrm{xx}^{\mathrm{e}}$ et $\mathrm{XxI}^{\mathrm{e}}$ siècles, particulièrement la peinture dans la période de l'après-guerre, en relation avec les écrits.
} 
La valeur de cet ensemble que nous appellerons ici « le manuscrit d'Orléans » tient d'abord au fait qu'il est entièrement de la main de Max Jacob, lequel a recopié son chef d'œuvre et l'a illustré de dessins et de gouaches. À la suite de cet article, le lecteur trouvera reproduit dans un encart couleur l'intégralité de ce manuscrit. Le « peintre d'images » qu'était aussi le poète, pour reprendre l'expression de Florent Fels $^{2}$, privilégiait dans ses lettres, on le sait, le terme dépréciatif de «salir »... On est tenté, bien au contraire d'écrire orner ou rehausser, voire l'antithèse même de « salir », enluminer. Mais c'est la magnifique reliure réalisée par le décorateur parisien Paul Bonet (1889-1971), artiste du livre, qui le rend plus précieux encore aux yeux des bibliophiles. Elle en fait un des fleurons de la Médiathèque d'Orléans qui l'a choisie pour représenter le xx $x^{\mathrm{e}}$ siècle dans son exposition de 2005, Dix siècles de reliures de la Bibliothèque municipale d'Orléans ${ }^{3}$.

Il est composé de 26 feuillets dont le tableau joint en annexe (p. 185-189) donne un descriptif détaillé. Sa dimension est de 36,5 x $25 \mathrm{~cm}$. Les pages, de formats variables, sont «montées en réserve en un volume in-folio relié box nuit aux plats entièrement mosaïqués ", pour reprendre une partie de la notice de la Médiathèque, elle-même redevable à la description du catalogue de l'Hôtel Drouot, qui l'a mis en vente ${ }^{4}$. Décrire ce manuscrit exceptionnel permettra de mettre en évidence un certain nombre des questions qu'il soulève. L'étude tentera de retracer d'abord son histoire, puis observera la reliure, en dialogue avec la page de titre, avant d'en venir aux illustrations, dans leur rapport au texte.

\section{$1990,1953,1943$}

Remonter le temps mène des repères les plus nets à des données plus incertaines. À la fin de l'année 1990, le manuscrit est acquis par la Médiathèque, ou plutôt, à l'époque, la Bibliothèque d'Orléans. Le manuscrit a été présenté dans la célèbre salle des ventes parisienne, l'Hôtel Drouot, une première fois, le 2 décembre 1989 (salle 9, commissaire-priseur Guy Loudmer). L'intitulé du lot dont il relevait alors (lot 215) nous apprend qu'il appartenait à une collection privée : Une Bibliothèque de connaisseur, $\mathrm{xx}^{\mathrm{e}}$ siècle, livres illustrés, éditions originales, manuscrits autographes, prestigieuses reliures. J'ai cherché le nom de ce « connaisseur» : probablement le collectionneur Jean Parizel, amateur de livres futuristes qui fut l'ami de Pierre-André Benoit ${ }^{5}$.

Le manuscrit n'ayant pas trouvé acquéreur, il repasse en vente le 27 novembre 1990 (Lettres et manuscrits autographes, experts Laurin, Guilloux, Buffetaud, Tailleur). L'extrait du catalogue, pour le numéro 151, confirme l'attention portée à la reliure, décrite au début de la notice ${ }^{6}$ : 
Le Cornet à dés: MANUSCRIT autographe signé avec DESSINS originaux. 26 feuillets (dont 14 recto-verso) la plupart montés en réserve en un volume in fol., relié box bleu nuit, aux plats entièrement mosaïqués, éclairés en leur centre d'une forme ovoïde ondulée du clair vers le bleu sombre, d'où fusent d'innombrables facettes mosaïquées en veau de toutes couleurs dans lesquelles les lettres du titre sur le premier plat à dominante jaune et rouge, et de l'autre sur le second plat à dominante jaune et verte, l'ensemble semé de valeurs de dés en cercles blancs mosaïqués, doublures et gardes de daim jaune d'or liseré de veau rouge, tranches dorées sur témoins, chemise demi-veau, étui (Paul Bonet, 1953).

Lors de la vente, le manuscrit est préempté par la Direction du Livre et de la Lecture, au profit de la Bibliothèque d'Orléans. Une délibération municipale ( $\mathrm{n}^{\circ} 20$, 21 déc. 1990) confirme la volonté de la Ville de s'en porter acquéreur pour la somme importante de 503832 francs et de faire une demande de subvention auprès du Ministère de la Culture qui répondra à la demande ; un courrier de Jack Lang, alors Ministre de la Culture, de la Communication, des Grands Travaux et du Bicentenaire, au Maire d'Orléans, Jean-Pierre Sueur, précise le montant de la subvention accordée, couvrant $50 \%$ de la somme, comme le souhaitait la Ville d'Orléans.

Passons à la deuxième date. C'est en mai 1953 que Paul Bonet achève la reliure du manuscrit, entreprise à la fin de l'année précédente. La première occurrence de cette date, la seule officielle concernant ce manuscrit, se trouve dans le précieux répertoire manuscrit du relieur lui-même ${ }^{7}$. Ce document précise la durée du travail (décembre 1952 - mai 1953) ainsi que les noms de deux des professionnels qui ont contribué à incarner les cartons de Paul Bonet : le relieur, spécialiste du cuir, René Desmules (1909-1978) qui a travaillé avec lui mais aussi avec d'autres artistes, dont Rose Adler ${ }^{8}$, ainsi que le célèbre doreur Raymond Mondange. Il révèle aussi que le manuscrit appartient à une " série ", le mot est de Paul Bonet lui-même, des six exemplaires du Cornet à dés (Desmules intervient sur les six). Ces reliures sont répertoriées par Bonet sous les numéros suivants : $\mathrm{n}^{\circ}$ 292, 1025, 1027, 1030, 1072 (exemplaire de la BnF, RES 8-NFZ-16, étudié en infra par Jean-Marc Pontier et Patricia Sustrac) et 1571. Or cinq des six livres sont des exemplaires de l'édition imprimée originale ${ }^{9}$. Voilà qui confirme l'intérêt du manuscrit d'Orléans, quatrième de la série (1030), entièrement réalisé à la main. Lisons la description qu'en fait Paul Bonet :

[Notice] 1030 M. JACOB Le Cornet à dés/Manuscrit établi par l'auteur avec 26 gouaches ou dessins / dans le texte. / Box bleu corbeau, c'est sur le ler plat le titre, sur le 2è le nom de l'auteur qui déterminent un jeu de lignes et de couleurs d'une composition quilpeut paraître abstraite encore qu'elle soit formée d'un centre ovalaire et d'un premier cadre pentagonal établi sur des tons orange puis 
d'un/second dans des tons rouges. Dans les intervalles et vers les bords jouent des bleus/sur le fond bleu - des valeurs de dés sont indiquées par des cercles blancs - le tout mosaïqué en veau; doublures et gardes : daim jaune./12-[19]52 - 5-[19]53 / Desmules, rel[ieur] Mondange dor [eur].

L'annotation en marge confirme l'aspiration de l'artiste-décorateur à poursuivre, de livre en livre, une même recherche, la résonance avec l'œuvre écrite : « Une de mes bonnes reliures où je crois être en accord avec Max Jacob et la période cubiste. »

À propos de l'exemplaire précédent, relié un mois avant celui qui nous intéresse (1027), Paul Bonet avait écrit en marge : « Recherches toujours incertaines vers un style Max Jacob. » Cette fois, il lui semble avoir atteint « l'accord » recherché avec le poète, associé ici au cubisme. C'est à la page de titre mais pas seulement à elle que son commentaire fait référence, on le verra.

Il reste à tenter d'élucider les conditions de réalisation de ce manuscrit par Max Jacob et revenir sur la date de 1943. Le manuscrit n'est accompagné d'aucune date, d'aucun appareil critique, d'aucun paratexte, en dehors de la date de la reliure et de la signature de Paul Bonet. La première question, la plus facile à résoudre, est celle du choix du texte : parmi les trois versions du Cornet à dés, c'est l'édition de 1922 qui a été recopiée, plutôt que l'édition originale de 1917 ou celle de 1923, la dernière publiée du vivant de l'auteur. Pourquoi ce choix ? Le poète n'avait-il plus d'exemplaire des éditions de 1917 ou de 1923 à sa disposition en $1943^{10}$. En tout cas, à ses yeux, l'édition de 1922, que Florent Fels avait établie à partir de celle de 1917, était précieuse ${ }^{11}$. À la différence de l'édition originale, on y trouve en particulier la préface antidatée de 1906 (O., 427), supprimée en 1923. En quelques mots, Max Jacob y affirmait l'originalité de sa conception du poème en prose, en se démarquant de Rimbaud, et à travers lui, de Pierre Reverdy. Elle ouvre le manuscrit d'Orléans. Il reste un point énigmatique : à l'édition de 1922, deux poèmes de l'édition de 1923 ont été ajoutés au feuillet 23 : « La mort morale » et « Rafraîchissons les vieux thèmes » (O., 401 et 412).

La deuxième question est celle du destinataire de ce manuscrit. A-t-il été commandé par Paul Bonet pour lui-même ou pour un collectionneur ? La comparaison de la notice de l'exemplaire $n^{\circ} 1030$ avec celle du $n^{\circ} 1072$ pourrait exclure la première solution : le $\mathrm{n}^{\circ} 1072$ est le recueil dédié par Max Jacob à Paul Bonet lui-même qui en a été le premier propriétaire ${ }^{12}$. Si le manuscrit d'Orléans $\left(\mathrm{n}^{\circ} 1030\right)$ lui avait été aussi destiné, ne contiendrait-il pas une dédicace en sa faveur ? Sans doute était-il destiné à un de ses clients, resté à l'époque anonyme. Il n'en demeure pas moins que neuf ans s'écoulent entre la mort de Max Jacob 
et la réalisation de la reliure (1944-1953), ce qui paraît un peu long. Cela pourrait t-il s'expliquer par les rationnements en matières premières de l'immédiate après-guerre qui ont pu ralentir les activités des relieurs ? Que s'est-il passé dans cette période ? L'exemplaire est-il resté entre les mains de Paul Bonet, avant son acquisition, probablement postérieure, par Jean Parizel ? Il reste à le découvrir.

Enfin, est-ce en 1943 que Max Jacob a élaboré le manuscrit ? Quatre des six exemplaires de Paul Bonet dont le manuscrit d'Orléans ont été reliés dans la même période (entre novembre 1952 et mai 1953) ; on pourrait en déduire qu'ils sont contemporains. Tout porte à croire qu'ils datent de 1943, date retenue sur le mode de l'hypothèse, par la Médiathèque d'Orléans. Examinons avec prudence les indices qui permettent de le penser. On connaît la situation tragique que subit alors le poète ${ }^{13}$. Le sort fait aux Juifs à partir de 1940, la pauvreté qui est la sienne, la disparition progressive de sa famille, tout lui impose ce moyen de subsistance, ce que confirme largement sa correspondance. Max Jacob n'a « rien le droit de publier $^{14} \gg$ depuis 1940. Il vit le plus discrètement possible, à Saint-Benoît-surLoire, d'où il demande à ses amis que le nom de Jacob n'apparaisse pas sur les enveloppes qui lui sont adressées ${ }^{15}$. Il peint, mais à l'automne, il est découragé du fait que ses gouaches s'écaillent ${ }^{16}$. En 1943, même s'il escompte encore quelques revenus de sa peinture ${ }^{17}$, les ouvrages enrichis de poèmes manuscrits ou de dessins et gouaches sont probablement sa principale ressource :

On m'envoie des exemplaires de ces livres et j'y mets quelques griffonnages ou des poèmes. Ça fait un millier de francs chaque fois : ça suffit. Espérons que ce truc durera jusqu'à la fin de mes jours ${ }^{18}$.

Plusieurs libraires lui en commandent : dans les correspondances citées en note, on trouve des allusions au libraire Maurice Rouam, 20 rue Jeanne d'Arc à Orléans ${ }^{19}$, à celui de Montargis ${ }^{20}$, à Julien Lanoë de Nantes ou à Claude Pascal (alias Monny de Boully) ${ }^{21}$. À ces noms, il faut évidemment ajouter Paul Bonet. Une lettre de Max Jacob à André Salmon, le 23 juin 1943, nous apprend que le relieur lui a passé plusieurs commandes cette année là :

Ma ressource est de "salir " des exemplaires de feues mes æuvres lesquelles, épuisées [...] valent 300 frs mais griffonnées en valent 3 000. J'ai dans ce genre d'exercice un charmant garçon, relieur, bon et lettré qui me gâte en billets de mille (un par volume mais payé d'avance); il s'appelle Paul Bonet ${ }^{22}$.

Le « charmant garçon » est à peine plus jeune que Max Jacob! Le ton de celui-ci où perce une certaine condescendance s'accorde sans doute à son agacement à l'égard du commerce du livre : Max Jacob déplore que la reliure 
plus que son travail assure le prix de l'œuvre ${ }^{23}$. Il éprouve pourtant respect et reconnaissance pour le relieur. Ces mots mêmes en portent la preuve.

La lettre à André Salmon constitue un des arguments majeurs en faveur de 1943 pour le manuscrit et les trois exemplaires imprimés reliés à la même période. En revanche, il faut distinguer le manuscrit de ces trois exemplaires : quand Max Jacob écrit à Marcel Béalu, quelques mois plus tard (le 13 octobre 1943) :

J'ai un exemplaire du Cornet à " salir » pour le relieur de luxe Paul Bonet. J'y passe mes journées : je lui recopie des tas de poèmes en prose dans les pages blanches et je lui fais des dessins ${ }^{24}$.

Il ne s'agit pas du manuscrit d'Orléans qu'il évoque, contrairement à ce que suggère André Cariou ${ }^{25}$, mais plutôt l'exemplaire imprimé n 1072 aujourd'hui propriété de la $\mathrm{BnF}$, dans lequel, comme il est précisé ici, les poèmes et les dessins sont placés dans les espaces laissées blancs par l'impression. On le comprend, beaucoup de questions demeurent sur l'origine du manuscrit.

\section{LA RELIURE DE PAUL BONET}

Les relations entre Max Jacob et Paul Bonet mériteraient une étude en soi. Le poète a eu connaissance du travail de Paul Bonet au début des années trente : celui-ci relie en 1930 Les Sept péchés capitaux illustrés par Chagall, livre auquel Max Jacob contribue ; puis, en 1933, le recueil édité par Maurice Sachs, Visions des souffrances et de la mort de Jésus fils de Dieu. Le premier Cornet à dés de la « série » de Paul Bonet est relié en 1935 (n²92) ${ }^{26}$. Et la bibliographie des ouvrages de Max Jacob reliés par lui s'étend bien au delà de 1944. En tout cas, les noms des deux artistes restent associés de la première édition posthume du Cornet à dés, " décorée d'après la maquette de Paul Bonet 》 publiée en 1946 par Gallimard, aux grandes ventes de livres aujourd'hui. Un grand spécialiste du livre a mis en évidence le sens et la valeur de leur entente dès 1963. Cette année-là, en effet, le célèbre administrateur de la Bibliothèque nationale, Julien Caïn (1887-1974), écrit la préface d'un catalogue d'exposition en hommage au relieur ${ }^{27}$. Les premières lignes citent une des dernières lettres de Jacob :

Le samedi 15 janvier 1944, quelques jours avant son arrestation qui devait le conduire à Drancy puis vers la mort dans un camp d'extermination, Max Jacob écrivait de Saint-Benoît-sur-Loire à Paul Bonet: "Tu n'as pas été peintre, tu es 
mieux! tu t'es fait une case bien spéciale dans l'art, tu as créé un art à toi, sans

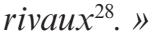

La référence au poète à l'entrée du catalogue est symboliquement très importante. C'est sur l'accord entre les deux artistes que Julien Caïn s'appuie ensuite pour définir l'art du relieur fondé sur la recherche des " correspondances », au sens de Baudelaire et de Swedenborg, entre le texte et la reliure, l'âme et le corps du livre en quelque sorte. Et de citer Paul Bonet lui-même :

La reliure de notre temps peut être une création originale, une création plastique qui reflète la personnalité de l'auteur. Ce n'est jamais une création gratuite; il lui faut d'abord rester soumise au livre...

Le lecteur qui a la chance de prendre en main le manuscrit d'Orléans se rend très vite à l'évidence : sous ses yeux s'incarne parfaitement l'équilibre si difficile à obtenir entre la liberté de la création et le respect du livre, mais aussi entre la sensibilité la plus fine et la maîtrise de l'architecture, sous l'égide de la géométrie, associée au cubisme.

Comment se manifestent ces correspondances, alors que la première impression est le contraste, d'abord déchirant, entre la précarité des pages manuscrites et la somptuosité de la reliure ? De deux manières, au moins. La première repose justement, et c'est un paradoxe, sur ce contraste. Accéder au manuscrit suppose l'accomplissement de plusieurs opérations successives : il faut d'abord prendre en main l'emboîtage bleu moucheté pour en sortir la chemise cartonnée du même bleu, puis ouvrir celle-ci. Alors seulement on peut sentir sous ses doigts le velouté du précieux « box bleu corbeau » et découvrir les deux plats, symétriques : le plat supérieur porte le titre, le plat inférieur le nom de l'auteur. À la fascination pour ces deux mosaïques, succède l'admiration pour le luxe du reste de la reliure : la matière vivante et les riches couleurs de ces « contreplats et gardes supérieures en daim jaune bordés d'un liseré de veau bordeaux portant le nom du relieur, Paul Bonet, et liseré de veau vert portant la date de la réalisation, 1953, pour le contreplat inférieur », ainsi que les tranches dorées. Les yeux se posent alors sur les pages de la main de Max Jacob : le lecteur songe à l'insistance du poète sur les salissures... Il mesure la mauvaise qualité du papier : les gouaches à l'eau ont pour support un papier trop fin (un papier à lettres, sans doute). Il aurait au moins fallu que le verso des illustrations reste vierge. Mais cela ne se produit qu'à douze reprises (sur 26), et encore de manière irrégulière. Le papier fin est utilisé au recto et au verso. Des pastels 
ou des gouaches au verso laissent des traces au recto. Ailleurs, on découvre un papier brun de mauvaise qualité collé sur du carton, du papier ligné, voire le verso d'une affichette d'une exposition à la Galerie Zak de Saint-Germain-desPrés. Au feuillet 3, un buste de moine au crayon apparaît à l'envers, au centre de la page, ce qui semble attester d'un réemploi du papier. Ajoutons que si les feuilles montées en réserve sont de format inégal (entre 35 et $22 \mathrm{~cm}$ de hauteur pour 25 à $14 \mathrm{~cm}$ de largeur), c'est du fait de leur hétérogénéité mais sans doute aussi parce que le relieur a été forcé de recouper des bords abîmés. Le poète manque cruellement de papier, même si la précarité de ce support n'est pas à surinterpréter pour autant. On fait le même constat à la lecture d'autres manuscrits de la Bibliothèque d'Orléans, Les Visions infernales (Ms 2263) par exemple.

L'effet de contraste entre le papier et la reliure est saisissant. On pourrait le comparer à celui qui, dans une église, saisit le fidèle face au tabernacle du maîtreautel révélant et dissimulant à la fois le pain considéré par le croyant comme le corps du Christ même. La reliure, loin de choquer par son luxe, pourrait jouer ce rôle de tabernacle : elle célèbre le prix spirituel de ce qui est caché ; elle cache et protège le secret de l'œuvre majeure du poète. À condition toutefois qu'elle soit en accord spirituel avec le texte. Et c'est bien le cas. Venons-en en effet à ces mosaïques de cuir qui répondent au titre du recueil et à la page de titre.

Les plats de couverture sont symétriques, l'un portant le titre, l'autre le nom de l'auteur. Les formes et les couleurs se répondent, en écho et en contraste, couleurs vives d'un côté, plus assombries de l'autre. Ils sont d'égale importance. À la différence de nombre de relieurs qui privilégient le premier plat, Paul Bonet a coutume de traiter à égalité les deux plats. Mais ici, la symétrie prend une dimension supplémentaire : le double oriente le regardeur vers les chiffres pairs qui dominent la composition. Et surtout ces deux formes oblongues répondent (en les enserrant) à celles peintes à la page de titre par Max Jacob. Il ne faut pas voir dans ces mosaïques la simple représentation des dés mais un jeu subtil qui propose au lecteur un mode de lecture. Expliquons-nous.

Le premier plat se présente comme des formes concentriques. Du centre aux bords, on trouve le jaune, l'orange, le rouge, séparés par des rubans bleus (le bleu qu'on retrouve sur le fond et l'emboîtage). L'orange, complémentaire du bleu, est placé entre les deux couleurs primaires dont il est le mélange. Au second plat, les formes sont identiques, mais inversées. Admirable logique du livre lui-même, dans le geste de le prendre dans les mains que cette position en 
miroir révèle ! Cette fois, les couleurs secondaires dominent : l'orange, le vert, le brun, séparés par les mêmes rubans bleus. Toutes les combinaisons de couleur se déploient donc, comme les chiffres des dés lancés, potentiellement ${ }^{29}$. Mais il y a plus : sur les deux faces du livre, chacune des couleurs se décompose, par le jeu des mosaïques, en 3 nuances ( 3 bleus, 3 rouges, 3 orange, 3 jaunes, etc.). 3 fois $4: 12$. Et ce subtil message chiffré se confirme si l'on se met à compter les cercles blancs qui rappellent évidemment les dés : les 6 positions de dés ne sont pas juxtaposées, ce serait trop démonstratif sans doute, mais, sur chacun des deux plats, en comptant les cercles, on arrive à 24 soit 2 fois 12 . Le relieur s'est amusé à ce clin d'œil à la numérologie qui fascinait le poète. On songe aussi à l'espoir toujours renouvelé au moment où l'on prend en main le cornet pour lancer les dés : celui de faire 12. N'allons pas plus loin car la légèreté importe. Mais ce jeu de couleurs et de formes pourrait être non seulement une illustration fantaisiste de ce dé-mot " sous toutes ses faces » qui signe la poésie de Max Jacob mais le désir de déclencher une lecture virevoltante, libre de ses mouvements. Les dés lancés tournent. Le regard du lecteur aussi qui, pour lire le titre et le nom de l'auteur de ces deux plats, doit faire mentalement pivoter le livre. Lecture souple qui n'est pas dérangée par l'hétérogénéité qui domine le manuscrit, mais l'accueille au contraire et se laisse surprendre. Lecture tournoyante, en réponse au geste même du peintre, évoqué très précisément dans un des poèmes du recueil :

Quand on fait un tableau, à chaque touche, il change tout entier, il tourne comme un cylindre et c'est presque interminable $(O ., 362)$.

Si l'art moderne est, depuis Apollinaire, sous le signe de la surprise, alors ce manuscrit en est l'exemple même, comme on va le voir. Et la reliure assure la fonction programmatique (Reverdy parlait de "fonction de portier ») que tient aussi, traditionnellement la page de titre. Chaque fois qu'il prend en mains ce manuscrit si subtilement relié, le plaisir du lecteur-regardeur se renouvelle, comme celui de l'enfant qui ouvre lentement la boîte contenant ses trésors rassemblés.

\section{« UN ARTISTE ASSISTÉ D’UN POÈTE »}

On le sait à présent, Max Jacob est peintre autant que poète. André Salmon, Florent Fels, aujourd'hui André Cariou et d'autres l'ont démontré30. Le lecteur se doit donc d'aller des textes aux enluminures et inversement, en considérant les 
deux expressions à égalité. Dans le cadre de cette étude, une analyse approfondie des échos entre elles est impossible. Après avoir donné quelques indications sur l'aspect technique de ce qu'on nommera des images par commodité, on tentera d'esquisser les lignes de force qui sourdent progressivement, derrière l'hétérogénéité apparente de l'ensemble.

\section{Comment Max Jacob a-T-IL « TRAVAILlé »?}

Se poser la question suppose de passer outre les dénégations du poète qui, en 1943, se refuse le titre de peintre :

Je ne peux pas appeler « travail» le métier de mettre des dessins sur des marges de mes livres comme je le fais tout cet hiver pour les uns et les autres ${ }^{31}$.

C'est aussi faire l'hypothèse qu'il prenait le plus grand soin à réaliser ses manuscrits. Certes, le degré d'application est très variable dans les vingt-huit images. Le passage rapide de gouache blanche sur la tête de l'âne de la Nativité 32 (9a) suppose beaucoup moins de soin que la belle gouache de la Descente de croix (10b). Mais l'écriture, soignée et posée, ne manifeste pas les pics d'émotion et les variations de rythmes sensibles dans le manuscrit des Visions infernales. Quant aux images, elles n'ont pas la spontanéité des esquisses, " traces-mouvements » qu'on trouve aussi dans ce second manuscrit : dessins nés de la rêverie, de l'ordre des surgissements spontanés, automatiques, analysés par le psychanalyste Serge Tisseron ${ }^{33}$.

Les images révèlent à la fois la simplicité des moyens utilisés et la pratique de techniques le plus souvent mixtes, même dans les dessins qui sont faits au crayon mais rehaussés à la plume. Les descriptifs du tableau, inspirés de ceux qui concernent Max Jacob au musée des Beaux-arts d'Orléans, le montrent. Max Jacob utilise le crayon noir, les crayons de couleurs (dont le fameux crayon de charpentier, rouge et bleu) et les pastels, la gouache passée au pinceau, le lavis (parfois mêlé de cendre de cigarette), enfin l'encre à la plume. On pouvait hésiter entre crayon noir et fusain, crayon gras et pastel. Jacob parle bien de fusain dans ses lettres mais il n'est pas toujours possible de distinguer dans les dessins l'usage d'un fusain de celui d'un simple crayon noir. Quant aux pastels, utilisés pour tracer des lignes ou pour réaliser des surfaces colorées au moyen d'un estompe (papier journal ou buvard sans doute) ou en mélangeant les pastels à de l'eau ${ }^{34}$, on sait par Roger Toulouse (dans son récit de sa dernière visite à Max Jacob, en 1944) que celui-ci a pu conserver ses pastels jusqu'à la fin : 
Bien vite, il recherchait les derniers poèmes de la semaine, et me montrait la difficulté qu'il avait eue à terminer le portrait d'Alfred Jarry qui séchait sur un coin de table, parmi les pastels, les cigarettes, le cendrier débordant d'allumettes, le livre de messe et une énorme cravate ${ }^{35}$.

Certaines images permettent de deviner le processus de réalisation : l'artiste semble d'abord dessiner au crayon, noir ou de couleur (rouge en particulier), posant ainsi les fondations du dessin ou de la gouache. Puis il traite les surfaces, par hachures, coloriage, estompe ou lavis. La plume vient à la fin pour donner relief et force à l'œuvre : certaines lignes sont tracées partiellement à l'encre, les autres se déduisent dans l'œil du spectateur qui les prolonge mentalement. La gouache (blanche, ou rouge pur pour le « Perroquet» du feuillet 13b) peut aussi rehausser le dessin, mais pas seulement. Il faut en effet accorder une place particulière à la gouache blanche, caractéristique du style de Jacob : elle est lumière opalescente ; elle porte la dynamique spirituelle dans le combat qui l'oppose au noir, comme celle implorée dans un des Derniers poèmes en vers et prose, «Taie divine » pour faire « reculer la myrrhe noire/ toute l'ancienne comédie/le jargon des monts/des démons/et leur lugubre fantaisie. » $(O ., 1564)$ :

La tache est descendue en blanc
nacrée comme un intérieur d'huître
je ne me vois que sous sa vitre.

L'ordre du processus de composition schématisé en 1989 par Roger Toulouse confirme ce qu'on observe dans le manuscrit ${ }^{36}$ :
Il s'y prenait en trois temps :
$1^{\circ}$ ) la construction du barème;
$\left.2^{\circ}\right)$ la pose de la gouache;
$\left.3^{\circ}\right)$ les rehauts à l'encre ou au crayon.

On constate ici l'insistance du peintre, conforme à ses propres préoccupations sur la construction de départ : "l'armature » qui peut même se faire à la règle et au compas comme dans la page de titre ou au feuillet $5 \mathrm{~b}$ ( A Athlètes et cerceaux »).

Mais la question la plus difficile à résoudre est celle de la composition. Peut-on aller plus loin que Francis Deguilly qui précise, en s'appuyant également sur Roger Toulouse, "Roger Toulouse qui vit Max Jacob réaliser cette copie, s'émerveillait de voir son ami enrichir son manuscrit de dessins plus ou moins anciens tirés de sa célèbre malle noire ${ }^{37} »$, ou qu'André Cariou qui parle 
de « dessins réutilisés ou composés pour la circonstance ${ }^{38} »$ ? L'artiste a-t-il enluminé des pages manuscrites, antérieures ? A-t-il écrit des poèmes dans les marges de dessins déjà réalisés, voire a-t-il sorti de sa malle des œuvres (les hors-texte) existantes? A-t-il créé ensemble textes et images, lui qui, jusqu'à la fin, élaborait « simultanément » gouaches et poèmes ${ }^{39} »$ ? Les différentes solutions coexistent probablement : les poèmes, « 1914 » et « La Guerre » (11a) semblent avoir été recopiés sur un dessin antérieur («Les Coqs couronnés »). La très belle gouache hors-texte, très travaillée dans un esprit cubiste, « Paysage avec citadelle » (12b), date peut-être de la fin des années trente, au moment où il échange avec Roger Toulouse, rencontré en 1936, sur la géométrie et l'architecture ; plus généralement, si, comme l'écrit Max Jacob, les gouaches « c'est de la méditation, six mois pour une ${ }^{40} »$, alors il est peu probable que les grandes gouaches hors texte aient pu être réalisées en 1943 alors que le peintre est forcé de multiplier ce qu'il appelle ses « griffonnages ». En revanche, la gouache du « Combat d'un moine avec un monstre » (6a) est simultanée du texte manuscrit. L'ensemble des poèmes et d'un dessin du perroquet (repassé sur les traits du perroquet au verso, 13a) également, peut-être. Par ailleurs, si le feuillet 21 ( « Adam et Ève ») est à l'envers, n'est-ce pas un signe patent d'une réutilisation du papier?

Se demander si le travail a été entièrement réalisé à Saint-Benoît-sur-Loire, si tous les dessins et peintures sont " originaux » stricto sensu ou s'il s'agit de copies importe moins que la réussite de l'ensemble qui vaut par son équilibre. Équilibre de funambule qui accorde sans peine apparente hasard et composition, le maître-mot du poète. Retrouver, au hasard des fouilles dans la malle, des pages manuscrites ou des dessins pour les ré-agencer impose de réaliser un nouvel équilibre dont un des signes évidents est le nombre équivalent de horstexte (14) et d'images dans le texte (13).

\section{DE L'HÉTÉROGÉNÉITÉ APPARENTE À L'ÉVIDENCE DE LA DYNAMIQUE UNITAIRE}

Les divers papiers et la mise en page donnent d'abord l'impression d'une grande hétérogénéité que confirme l'observation des images, variées, voire apparemment contradictoires par leur esthétique et la diversité de leur origine. On est tenté de les classer d'abord en trois groupes : 1. Les œuvres d'inspiration cubiste, 2. Les sujets classiques, 3. Les scènes religieuses. Au premier groupe appartiennent le titre (1a), des paysages ( $12 \mathrm{~b}$ et $23 \mathrm{~b}$ ), des figures ou des formes $(5 \mathrm{~b}, 7 \mathrm{~b}, 11 \mathrm{a})$. Au deuxième, des scènes de genre $(17 \mathrm{a}$, la scène de ballet $2 \mathrm{~b}$ et l'étrange navire $4 b$ ), des portraits d'hommes (15b), des animaux (13a et b, 19a, 25a). Le Bonnet d'âne souvenir de Radiguet (18a) est aussi burlesque que la scène de ballet. Quant aux sujets religieux, ils forment l'ensemble le plus important. 
Plusieurs scènes de la Bible sont représentées : « La Fuite en Égypte » (8a), «La Nativité » (9a), "La Descente de croix » (10b), " Adam et Ève chassés du paradis » (21b), " Le Baiser de Judas » (24a) et peut-être « La Visite de l'ange à Joseph » (25b). Mais on trouve aussi des dessins de religieux (3a et $3 b$ ) ; des anges, des personnages féminins, des martyrs avec palmes, " le Combat d'un moine avec un monstre « (6a), personnages apparemment inspirés de peintures romanes (14b, 16a, 20a) jusqu'à la fantaisie finale de « La Conférence tenue à Walpurgis du haut d'une vache fiscale » (26b).

Ce classement, pourtant, se révèle vite inopérant, tant la dynamique unitaire à l'échelle des motifs, de l'esthétique, mais aussi du processus de création est puissante. Limitons la démonstration à quelques exemples, en commençant par les motifs. Les motifs animaliers, appartenant ordinairement à la scène de genre, relèvent, chez Jacob, de l'inspiration religieuse. Qu'on songe à la prégnance de l'Arche de Noé dans son œuvre peinte, - comme dans les chapiteaux et les peintures romanes ${ }^{41}$. "Le Grand cerf » (19a) dont les bois imposent à la page sa composition verticale pourrait être l'envoyé de Dieu de la légende de saint Julien L'Hospitalier, le motif de la conversion revenant sans cesse dans l'œuvre et prenant un relief évident à la période qui nous intéresse, alors que le poète est menacé en tant que juif. Les Coqs couronnés, représentent l'Église, si l'on se réfère aux Méditations sur le chemin de croix :

\section{Le coq est l'animal de la gloire et voici l'Église caractérisée (O., 1493).}

Mais la proximité du poème « La Guerre » ouvre à une autre interprétation, en parallèle. « Le Perroquet » (13a), qui reprend un détail d'un lavis, L'Homme à la faux, peint vers $1936^{42}$, est une figure christique, par exemple dans le retable de Giovanni Bellini, à Vicence, Le Baptême du Christ (1500-1502) où il est placé aux pieds du Christ. La conversion, encore. Mais les animaux du bestiaire du peintre ont aussi bien souvent son double, tel le perroquet du lavis, accompagnant un autoportrait satirique du poète, ou cette figure animale au monocle, à peine visible dans la dernière image (26b), très proche à la fois des pélicans et du babouin ${ }^{43}$ de l'exemplaire 1072, portant cette légende «Seul véritable portrait de l'auteur ». La dérision, inscrite fortement dans l'esthétique du poète, qui prône «l'acide, l'aigre », contre « le raplapla bourgeois ${ }^{44}$ », est pourtant indissociable de l'humilité du pécheur dont témoigne une autre figure récurrente dans le manuscrit, celle de l'âne, présent dans les grands moments de la légende christique, de la Nativité à la Fuite en Égypte ou à l'entrée à Jérusalem, et autre double du poète. Le double et sa multiplication permise par le bestiaire (le 2, chiffre pair), 
pourrait être une des clés du manuscrit. Rien de contradictoire dans ces tonalités que d'autres opposent et qui, ensemble, constituent la signature du poète-peintre.

Ensuite, la cohérence esthétique est particulièrement sensible ici, même si, comme je l'ai démontré ailleurs ${ }^{45}$, elle caractérise toute l'œuvre. C'est bien par ses images, et pas seulement par ses textes, ou plutôt dans l'accord des deux, que le manuscrit du Cornet célèbre la modernité des avant-gardes cubistes, au-delà de la page de titre. Par le traitement des motifs religieux, Max Jacob s'inscrit en faux contre l'art saint-sulpicien, lui qui fut partisan du renouveau de l'art religieux lancé, en 1919, par les « Ateliers d'art sacré » de Maurice Denis et Georges Desvallières, rejoints par le père dominicain Marie-Alain Couturier $^{46}$. On sait qu'il admirait Maurice Denis, comme le révèle, parmi d'autres témoignages, la lettre qu'il adresse à Jean Follain, lors du décès accidentel de son beau-père, le 19 novembre 1943. La modernité de Maurice Denis reprend, écrit-il dans cette lettre ${ }^{47}$, « la tradition médiévale ». De fait, la gouache de « la Descente de croix » (10b) est inspirée à la fois de celle de Denis et de la peinture romane. À partir de la fin des années trente, trois raisons ramènent Max Jacob vers cette tradition dans sa relation avec la modernité, la première étant sa parfaite connaissance des chapiteaux de la tour-porche et de la croisée des transepts de l'abbatiale de Fleury. On sait que Max Jacob, quand il faisait visiter la tour-porche, montrait les figures romanes en disant que Picasso tentait de faire la même chose. La seconde est les nombreux échanges sur l'architecture avec le jeune peintre Roger Toulouse fasciné par le cubisme. Lors de sa première visite à Max Jacob, en 1936, Toulouse a vu sur la table le livre de Matila Ghyka Le Nombre d'or (1931) et affirme que «toutes les compositions du peintre " reposent sur lui ${ }^{48}$. " Les compositions cubistes citées plus haut, ainsi qu' "Athlètes et cerceaux » (5b), proche d'une étude à la plume de 1938, intitulée Athlète grec, et la Visite de l'ange à Joseph, sont à l'évidence construits selon son principe. La troisième est la découverte, en 1940, par l'entremise de Fernand Léger ou de Jacques Lipchitz, du livre illustré d'Henri Focillon, Peintures romanes des églises de France (1938), qui provoque l'enthousiasme du poète partagé avec son ami Daniel-Henry Kahnweiler, le théoricien du cubisme. Max Jacob voit dans cette forme artistique non seulement le contrepoint à «l'art Saint-Sulpice » mais aussi un fondement important de l'art moderne ${ }^{49}$. De même, le peintre Georges Braque opposait «l'art de composition » du Moyen-âge à « l'art d'imitation » (ou, disait-il, de « mise en scène ») de la Renaissance ${ }^{50}$. On ajoutera que la conception même du manuscrit relève de la modernité : en effet, textes et images se juxtaposent, sans hiérarchie aucune et surtout sans que jamais on puisse attribuer aux images 
une fonction d'illustration (autrement dit de subordination des images au texte). On retrouve là le principe de l'esthétique des premiers livres de peintre, ceux édités par Kahnweiler, justement, dès $1909^{51}$. Précisons cependant pour conclure qu'aux yeux de Max Jacob comme de Reverdy, le cubisme n'est pas pour autant à confondre avec " les mathématiques ${ }^{52}$ " : l'émotion y est reine. Cet aspect essentiel ne peut malheureusement être développé ici.

Le troisième point sur lequel il faut interroger la dynamique unitaire est celui du processus de création. Il est difficile de démontrer qu'il est identique pour les textes et les images. Il serait d'ailleurs imprudent de comparer l'écriture de 1917 aux images de 1943, dont l'état d'achèvement est extrêmement variable, du simple croquis à la gouache élaborée, et ce, même si le peintre-poète a toujours insisté sur la cohérence de l'ensemble de son œuvre. Il y a en revanche une grande ressemblance entre le geste de copier les poèmes que le poète assume totalement, même si la situation tragique les rejette dans le passé de la jeunesse, et celui de copier les images. Les deux sont de l'ordre de la rumination (au sens religieux du terme) et du rassemblement, fondé sur la réminiscence. Les images surgissent, au fil de la copie du texte ou dans l'inspiration du moment, comme surgissent, vivantes, les figures sur les chapiteaux de la tour-porche, à la fois insolites et sans logique apparente, mais nées de modèles repris, copiés ou transformés par les artisans sculpteurs qui circulaient d'église en église. Loin d'être originales, la plupart de ces images viennent d'ailleurs (empruntées qu'elles sont à d'autres artistes et en tout cas au passé de peintre de Max Jacob). Un travail minutieux d'archéologie permettrait de retrouver les sources et les transformations de ces images. Ainsi la Visite de l'ange à Joseph (25b) ressemble étrangement à un dessin des Méditations sur le Chemin de croix, si ce n'est que le personnage qui attend le visiteur y est une femme et non un homme. Il y a reprise et variation à la fois, comme dans le texte même des Méditations, inspiré librement de la méthode de saint François de Sales, toujours sur le même thème mais renouvelé par le souvenir, l'image ou l'émotion de l'instant. Les dés qui roulent se mettraient-ils à symboliser le temps spirituel qui est cyclique?

Pourquoi parler de rassemblement ? Le manuscrit n'a pas la solennité tragique d'un testament, en raison des écarts de traitement entre les images, des effets de surprise ou de la fantaisie qui règne jusqu'à l'énigmatique croquis final, Conférence tenue à Walpurgis du haut d'une vache fiscale : cette image, même si elle est à nos yeux lourde de sens ${ }^{53}$, burlesque en diable, répond en effet plutôt à l'esthétique décrite par Jean Cocteau : 
Tout chef d'œuvre est fait d'aveux cachés, de calculs, de calembours hautains, d'étranges devinettes ${ }^{54}$.

Mais le manuscrit vibre. Nous sommes des «êtres vibrants », répète le poète. Il vibre de ce qu'il paraît rassembler, dans les derniers jours de la vie d'un homme lucide sur la proximité de l'issue, les seuls trésors qu'on ne peut lui prendre et sur lesquels il se concentre contre l'adversité : ces mots et ces images qui l'habitent ne sont pas seulement des témoins; ils ne se résument pas à des restes, rassemblés au hasard. Ils sont tout cela ; mais, dans leur diversité apparente, dans leur énigmatique simplicité, dans leur relation en tension, ils font æuvre ensemble. La phrase magnifique écrite à André Salmon, "Les peintres cubistes connaissent les paraboles et n'en calculent pas la marche algébrique $\|^{55}$ révèle l'accord entre modernité et émotion, hasard et composition, « art de création » et fable qui est au cœur même du Cornet à dés. La pratique religieuse de Jacob ne vient pas le détourner de la voie explorée à partir de 1898 et dont témoigne la première l'édition de 1917. Elle vient au contraire en déployer encore le contenu. Et ce sont les images du manuscrit qui le révèlent, elles qui sont venues s'installer dans les «marges de silence » générées par le poème en prose.

\section{NOTES}

1 MO, Réserve précieuse, ms autographe 2541. Nous remercions M. Francis Deguilly, ancien directeur de la Bibliothèque Municipale d'Orléans pour ses recherches et Mme Patricia Sustrac pour son aide, les conclusions de ses recherches et les documents transmis lors de la rédaction de cet article.

2 Dans le $\mathrm{n}^{\circ} 2$ de la revue belge, Le Disque vert, consacré à Max Jacob (nov. 1923), l'article de Florent Fels, intitulé « Max Jacob, peintre d'images » (p. 26-28) est le deuxième entièrement dédié à l'activité de peintre du poète, le premier étant le chapitre que lui consacre André Salmon dans ses Propos d'atelier (Crès, [janvier] 1923, «L'atelier du frère Mathorel » (O., 71).

3 BOSC Aurélie, MAIGNAUT Marie, MARION Michel et MONGINOUX Anne, Dix siècles de reliures de la Bibliothèque municipale d'Orléans [exposition, 17 septembre-17 décembre 2005], Orléans : B. M. d’Orléans, 2005, p. 138-139. La notice précise que la Bibliothèque possède aussi deux autres ouvrages de Jacob reliés par Paul Bonet, Le Cabinet noir et Méditations religieuses.

4 Vente du 27 novembre 1990, $\mathrm{n}^{\circ}$ 151, Hôtel Drouot.

5 Jean Parizel est « le principal collectionneur des livres de PAB» (CORON Antoine, PAB : Le Fruit donné : BnF, 1989, p. 54). Dans ce catalogue d'exposition, est reproduite une photographie prise par P.-André Benoit, en 1962, chez lui, de Jean Parizel en compagnie de Gianni Bertini, p. 44.

6 Cette première description est suivie d'une présentation de l'œuvre de Jacob et d'un inventaire du contenu de chaque feuillet, poèmes et images (titrées par Max Jacob ou par les experts, et décrites 
du point de vue de la technique utilisée et du motif représenté). Bien que détaillées, il manque à cette description des précisions ajoutées dans notre tableau ( $c f$., infra Annexe).

$7 \quad C f$. l'article de Jean-Marc Pontier et Patricia Sustrac, n. 10.

8 On trouve des indications concernant René Desmules sur le site de la librairie parisienne Fata Libelli.

9 Dans les ex. 292 et 1027, Jacob a ajouté des poèmes manuscrits ; dans l'exemplaire 1072, des poèmes et des dessins.

10 En 1940, Jacob a donné plusieurs ex. du Cornet, toutes éditions confondues, à la Bibliothèque de Quimper pour constituer son fonds ( $c f$. supra l'article de Patricia Sustrac " Liber amicorum... »), en 1942, il offre son ex. de 1917 à Monny de Boully (Ibid.). Par ailleurs, nous ignorons le sort du stock de l'édition originale du recueil ( $c f$. infra « Brève histoire du recueil »).

11 Cf. supra l'article de Patricia Sustrac « Brève histoire du recueil ».

$12 C f$. l'article de Jean-Marc Pontier et Patricia Sustrac.

13 Voir SUSTRAC Patricia, " La mort de Max Jacob : réalité et représentations. Étapes des persécutions contre Max Jacob et sa famille 1940-1944 », CMJ, n 9, 2009, p. 103-127 et LEROY Géraldi, "Max Jacob sous 1〉Occupation dans sa correspondance », CMJ, n 13-14, 2013, p. 139-155.

14 AAIII, 79, lettre à Jean Colle, 4 déc. 1943.

15 La prudence de Jacob pourrait expliquer l'absence de date dans le manuscrit. Quant au commanditaire, si tant est qu'il y en eût un, il est sans doute resté ignoré de lui.

16 « Je n'ai plus le courage de peindre, parce que la gouache se fendille et que je ne peux pas vendre de la camelote pas solide ; quant à l'aquarelle, tu sais que ça ne se conserve pas en couleurs. » (AAIII, p. 67, lettre à [R. Arnal], 9 octobre 1943). Une autre lettre du 12 septembre 1943 faisait le même constat ( $M J A S$, p. 320). Le 28 octobre, Max Jacob explique que Roger Toulouse lui offre des cartons qui devraient résoudre le problème (ibid., p. 325).

17 Le 7 juillet 1943, Roger Toulouse emporte des peintures de Max Jacob pour les présenter à Paris chez Drouand-David (ibid., p. 314).

18 AAIII, 67, lettre à [R. Arnal], 9 oct. 1943.

19 AAIII, 72, lettre à François Garnier.

20 Lettre à André Salmon, 8 mai 1942, MJAS, p. 276.

214 juin et 29 octobre 1941, ibid., p. 234, 252, 300. Lettre à Roger Toulouse, 24 mars 1943 (JACOB Max, Lettres à Roger Toulouse, 1937-1944, correspondance présentée et annotée par Patricia Sustrac et Christine Van Rogger Andreucci, Troyes : Les Cahiers bleus, 1992, p. 89).

22 Lettre à André Salmon, 23 juin 1943, MJAS, p. 311.

23 «Le Phanérogame a fait 4500 frs à l'Hôtel à cause d'une reliure de Paul Bonet. C'est absurde!», lettre citée par A. Monginoux, op. cit., p. 139.

24 A. Monginoux cite une autre lettre en se demandant si elle peut concerner le manuscrit : "J'ai illustré un exemplaire du Cornet à dés pour un relieur bibliophile, Paul Bonet ». Il semble que non, pour les mêmes raisons.

25 Cf. CARIOU André, Le Peintre inavoué, Spézet : Coop Breizh, 2014, p. 91.

$26 C f$. infra l'article de Jean-Marc Pontier et Patricia Sustrac, n. 12.

27 Bibliothèque reliée par Paul Bonet, Reliures mosaïquées sur très beaux livres illustrés, éditions originales et de luxe, grands textes classiques. Préface de Julien Caïn, introduction de P. Bonet, Librairie Marcel Sautier, 1963 (catalogue d'exposition).

28 Cette lettre truffe l'exemplaire de La Côte, relié par P. Bonet, BnF RES-P-YN-4.

29 Toutes les études sur Paul Bonet confirment sa grande connaissance de l'œuvre de Jacob. Mon commentaire est évidemment partiel. On peut penser que les allusions subtiles à l'homme 
et à l'œuvre se multiplient dans les reliures. Ainsi, Patricia Sustrac rappelle que le bleu et le jaune sont les couleurs du Christ lors de sa première apparition $(O ., 471)$. Ces couleurs sont également dominantes dans la faïence de la ville de cœur de Max Jacob : Quimper.

30 La formule de titre est empruntée à André Salmon, dont le texte se trouve à l'entrée du catalogue Max Jacob peintre, Paris-Pont-Aven : Galerie de la Poste, mars-avril 1988, p. 19. Parmi les études, outre les écrits d'André Salmon et de Florent Fels déjà signalés, il faut citer les nombreux travaux d'André Cariou, conservateur et ancien directeur du musée de Quimper (dont le catalogue, signé avec Hélène SECKEL, Max Jacob et Picasso, 21 juin-4 septembre 1994 : RMN, 1994), ainsi que l' introduction à Lettres à Roger Toulouse : 1937-1944, op. cit, de Christine Van Rogger Andreucci.

31 Lettre du 15 déc. 1943 à Roger Toulouse, op. cit., p. 95.

32 Le parti a été pris de ne mettre en italiques que les images dont le titre a été donné par Max Jacob lui-même, en marge des images. Les autres titres (ceux du catalogue de l'Hôtel Drouot, parfois modifiés dans notre tableau) sont seulement signalés par une majuscule initiale.

33 TISSERON Serge, Psychanalyse de l'image, Dunod, 1995.

34 « Max Jacob trempait volontiers (ses pastels) dans l'eau, qu'il sucrait pour les écraser sur le papier », André Salmon, dans Max Jacob peintre, op. cit., p. 23.

35 « La dernière fois », Roger Toulouse cité par Jean-Louis Gautreau, « Max Jacob est arrêté, histoire d'une amitié fidèle au-delà de la mort ", Revue des Amis de Roger Toulouse, n 4, sept. 1999, p. 49. Les pastels qu'a vus Toulouse sont probablement ceux qu'il a ensuite donnés au Musée d'Orléans.

36 Roger Toulouse, « Max Jacob peintre, interview de Roger Toulouse par René Franck », Les Amis du Musée des Beaux-Arts d’Orléans, nº 8, déc. 1989, p. 2.

37 DEGUILLY Francis, «Le fonds jacobien de la bibliothèque municipale d'Orléans » dans ALBERT-BIROT Arlette (dir.), Max Jacob et la création : colloque d'Orléans, J.-Michel Place, 1994, p. 232.

38 CARIOU André, Le Peintre inavoué, op. cit., p. 90.

39 «Il avait [...] toujours sur sa table de travail une gouache et un poème qu'il élaborait simultanément », dans « Max Jacob peintre, interview de Roger Toulouse par René Franck », art. cit., p. 3 .

40 Lettre à Roger Toulouse, [1937], op. cit., p. 14.

41 L'appartenance des animaux au monde religieux est une constante de la peinture ou de la sculpture romanes comme de l'art de la Renaissance, en Italie ou en Flandres.

42 L'Homme à la faux est reproduit dans le catalogue : CARIOU André \& KLINKA Isabelle, Max Jacob, portraits d'artistes : [Exposition Musée des Beaux-arts de Quimper, 11 septembre-28 novembre 2004, Musée des Beaux-Arts d'Orléans, 11 décembre 2004 - 13 mars 2005], Somogy, 2004, p. 134.

$43 C f$. l'article infra de Jean-Marc Pontier et Patricia Sustrac et $O ., 1534$.

44 AAIII, 71, lettre à [R. Arnal], 28 oct. 1943.

45 NICOL Françoise, « Max Jacob, peintre », dans LARDOUX Jacques (dir.), Max Jacob et l'école de Rochefort, Angers : PU d'Angers, 2005, p. 109-123. L'étude, dont une partie seulement a été retenue, mettait en relation inspiration cubiste et art roman, dans la proximité de Roger Toulouse, et se fondait sur l'idée que Max Jacob est peintre, tout autant que poète.

46 La gouache du manuscrit Méditations sur le chemin de croix ( $\mathrm{ms} 2589,1939)$ de la Médiathèque d'Orléans, intitulée "Art Saint-Sulpice ", témoigne de la mise à distance de l'esthétique désincarnée et convenue répandue dans les églises, à la fin du XIX ${ }^{\mathrm{e}}$ siècle.

47 AAIII, 75, lettre à Jean Follain. 
48 « Max Jacob peintre, interview de Roger Toulouse par René Franck », art. cit., p. 3.

$49 \mathrm{Au}$ même moment, Max Jacob découvre Fra Angelico (vers 1400-1455). La Nativité du manuscrit (9a) est inspirée de celle du peintre toscan.

50 Georges Braque, « Le Jour et la Nuit », Cahiers de Georges Braque, 1917-1952, Gallimard, 1952, p. 40.

51 À l'heure où le cubisme rompt l'attache qui lie étroitement figuration et représentation, le livre se libère du joug de l'illustration. Rappelons que le livre de Max Jacob publié par la galerie Simon (Kahnweiler), Saint Matorel, illustré de quatre eaux-fortes hors-texte par Pablo Picasso, date de 1911.

52 Lettre à André Salmon, 23 avril 1943, MJAS, p. 305.

53 Le poète connaissait certainement le poème de Verlaine et bien sûr le Faust de Goethe, mais probablement pas une des premières dénonciations du nazisme par le satiriste Karl Kraus, La troisième nuit de Walpurgis (1933), pourtant on ne peut qu'y songer aujourd'hui. Quant à la "vache (à lait ?) fiscale ", fait-elle allusion aux ennuis du poète avec le fisc lors de la reconstitution de son patrimoine en 1929 en vue de compléter son dossier de demande d'indemnisation après son accident de la route (MOUSLI, 363) ?

54 COCTEAU Jean, Essai de critique indirecte, Grasset, 1932, p. 86. On peut commenter par ces lignes la gouache Bonnet d'âne souvenir de Radiguet (18a). Légère, elle fait évidemment allusion à un épisode de la vie de Jacob (et de Cocteau). Et le bonnet en question se révèle très proche de celui porté par le personnage du Fou ou Arlequin de bronze de Picasso (1905), reproduit dans le catalogue Max Jacob, portraits d'artistes, op. cit., p. 29.

55 Lettre à André Salmon, 23 avril 1943, MJAS, p. 305.

\section{NOTE DE LECTURE DE L'INVENTAIRE SCIENTIFIQUE}

26 feuillets. $-\mathrm{a}-:$ recto et $-\mathrm{b}-:$ verso. Le feuillet $1 \mathrm{~b}$ est face au feuillet $2 \mathrm{a}, 2 \mathrm{~b}$ face à $3 \mathrm{a}$, etc.

En gris : recto vierge du feuillet. Dans les autres cas, texte et dessins sont au recto et au verso du même feuillet, constitué généralement d'un papier blanc, fin (pb).

On trouve 28 illustrations : la page de titre, 14 illustrations hors-texte (HT), en pleine page et 14 illustrations dans le texte (in-T). Les titres des illustrations entre crochets ne sont pas de Max Jacob. Ils sont partiellement empruntés au descriptif du catalogue de la vente du 27 novembre 1990. Seuls sont de Max Jacob les titres entre guillemets.

Pour les textes, la règle appliquée est similaire : les titres entre crochets ne sont pas recopiés mais apparaissent dans les éditions imprimées. Quant aux titres suivis de points de suspension (par exemple : «Tu sors...»), ils correspondent non à des titres isolés typographiquement mais à l'incipit des poèmes. 


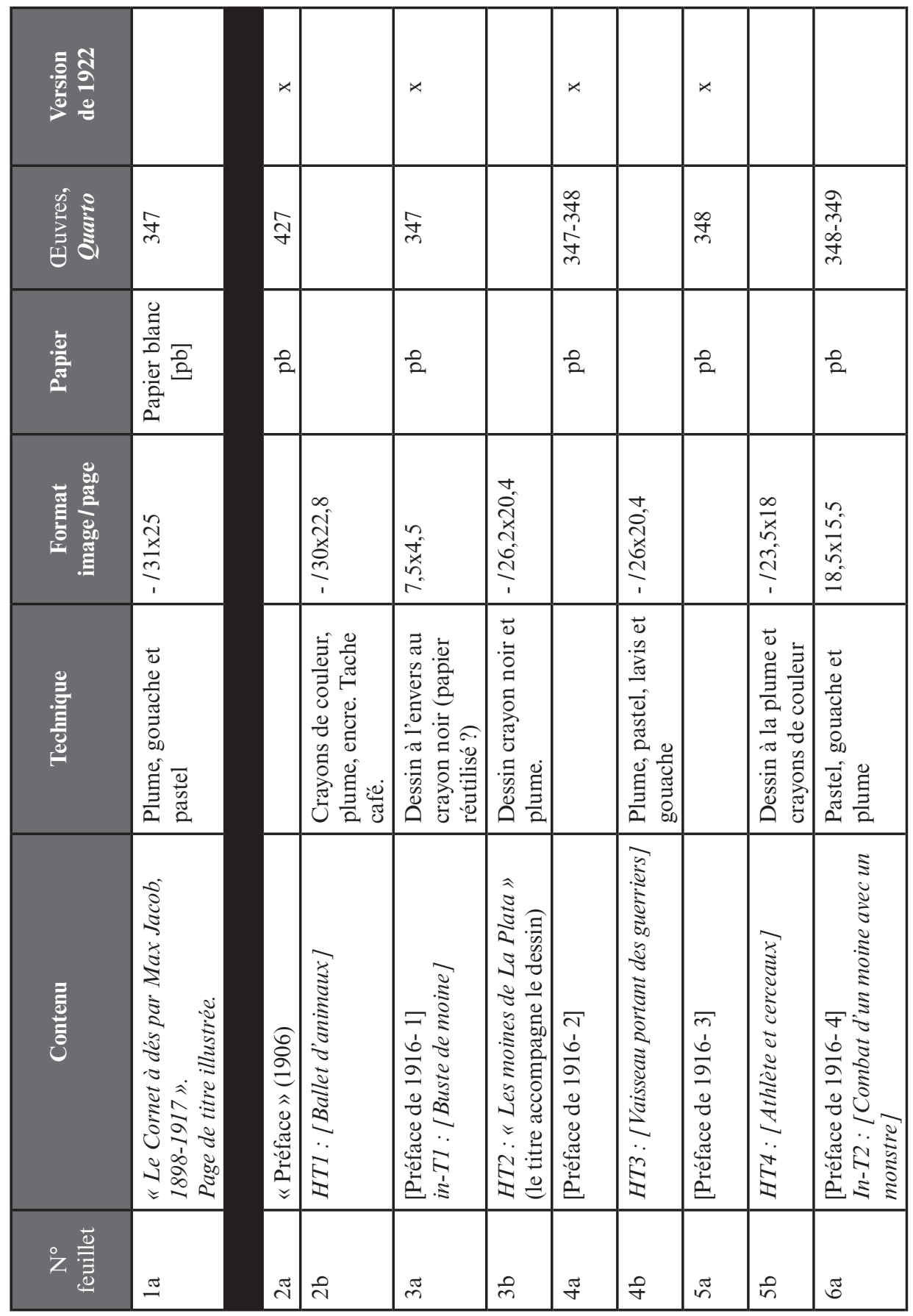




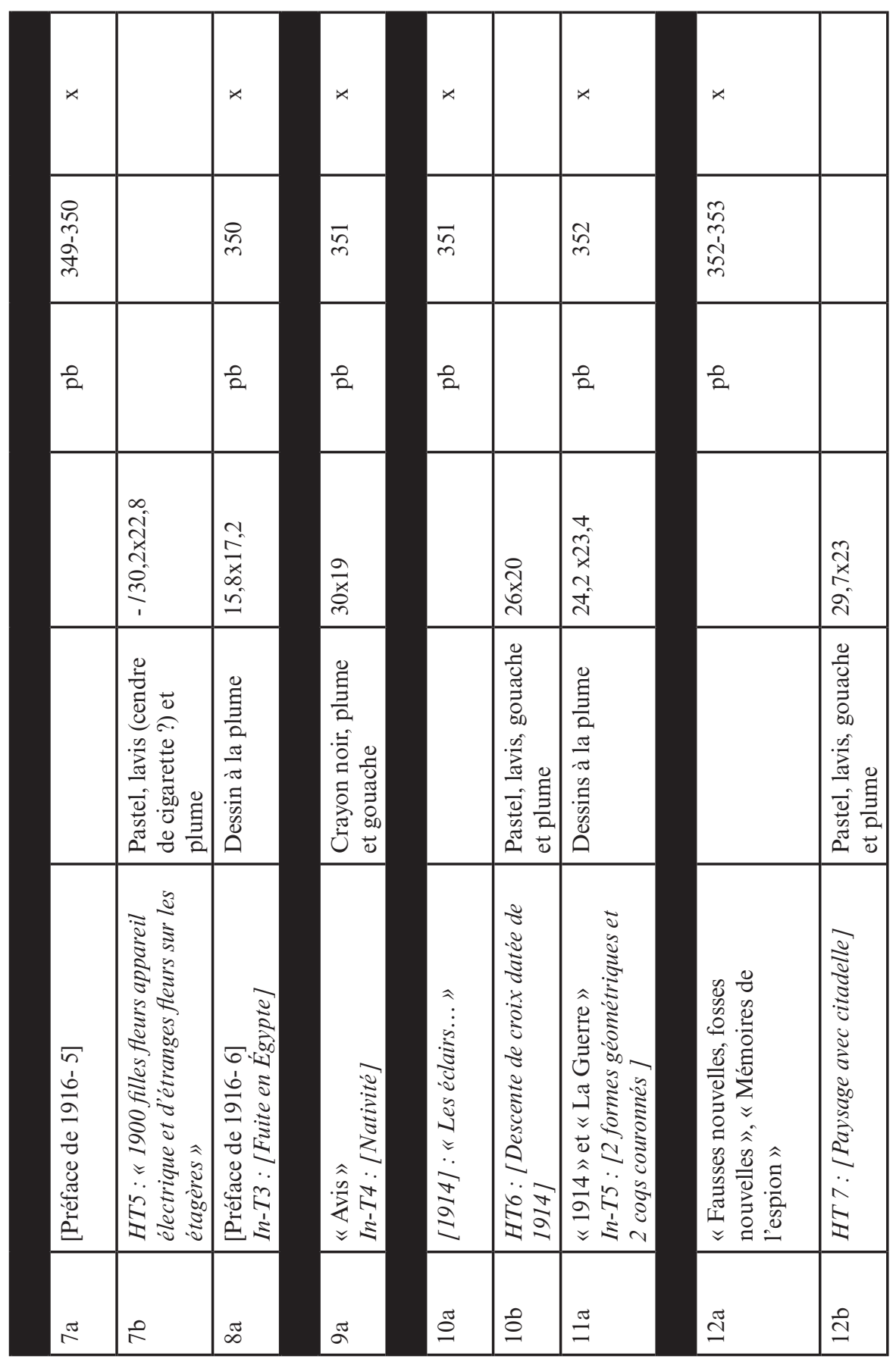




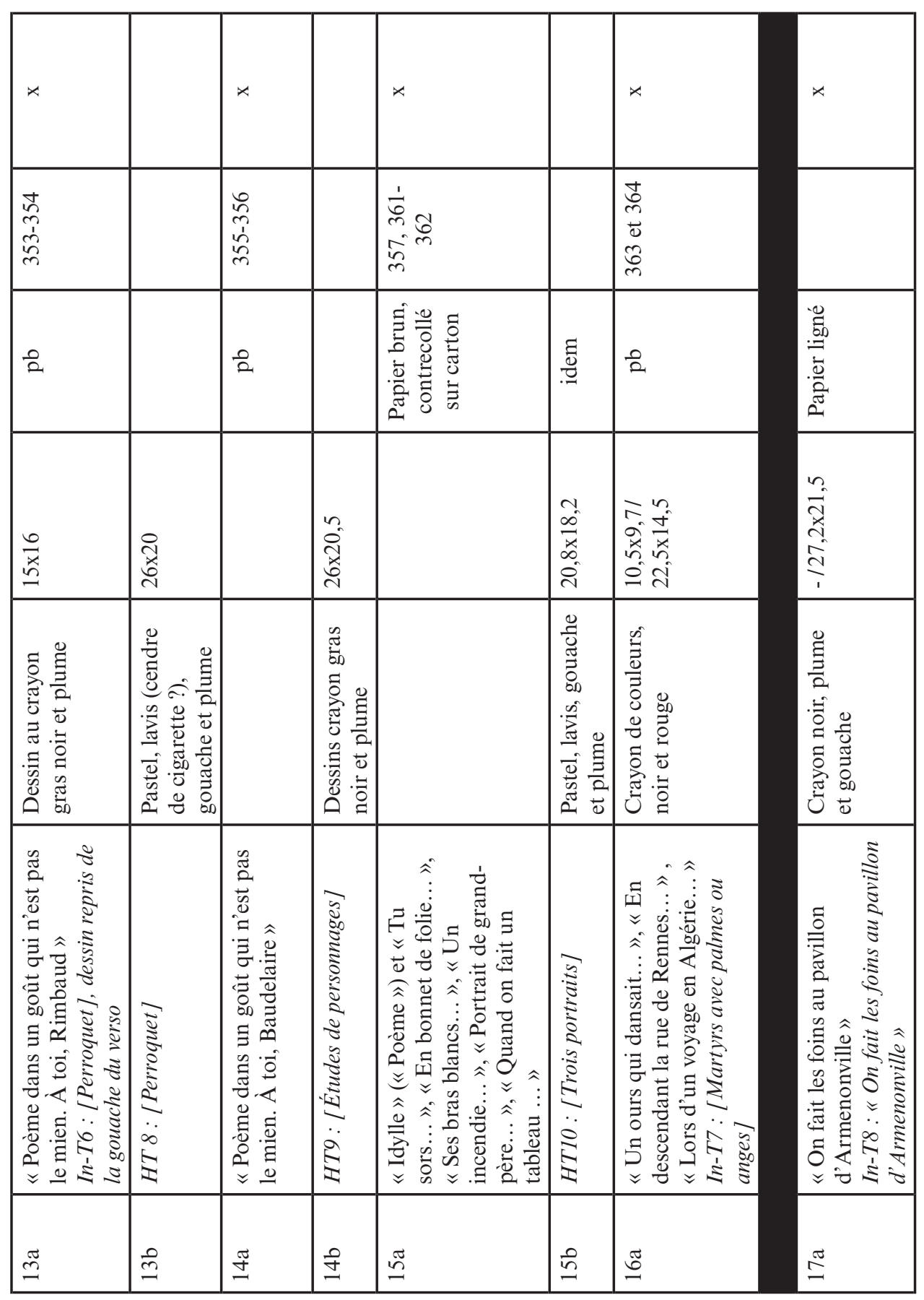




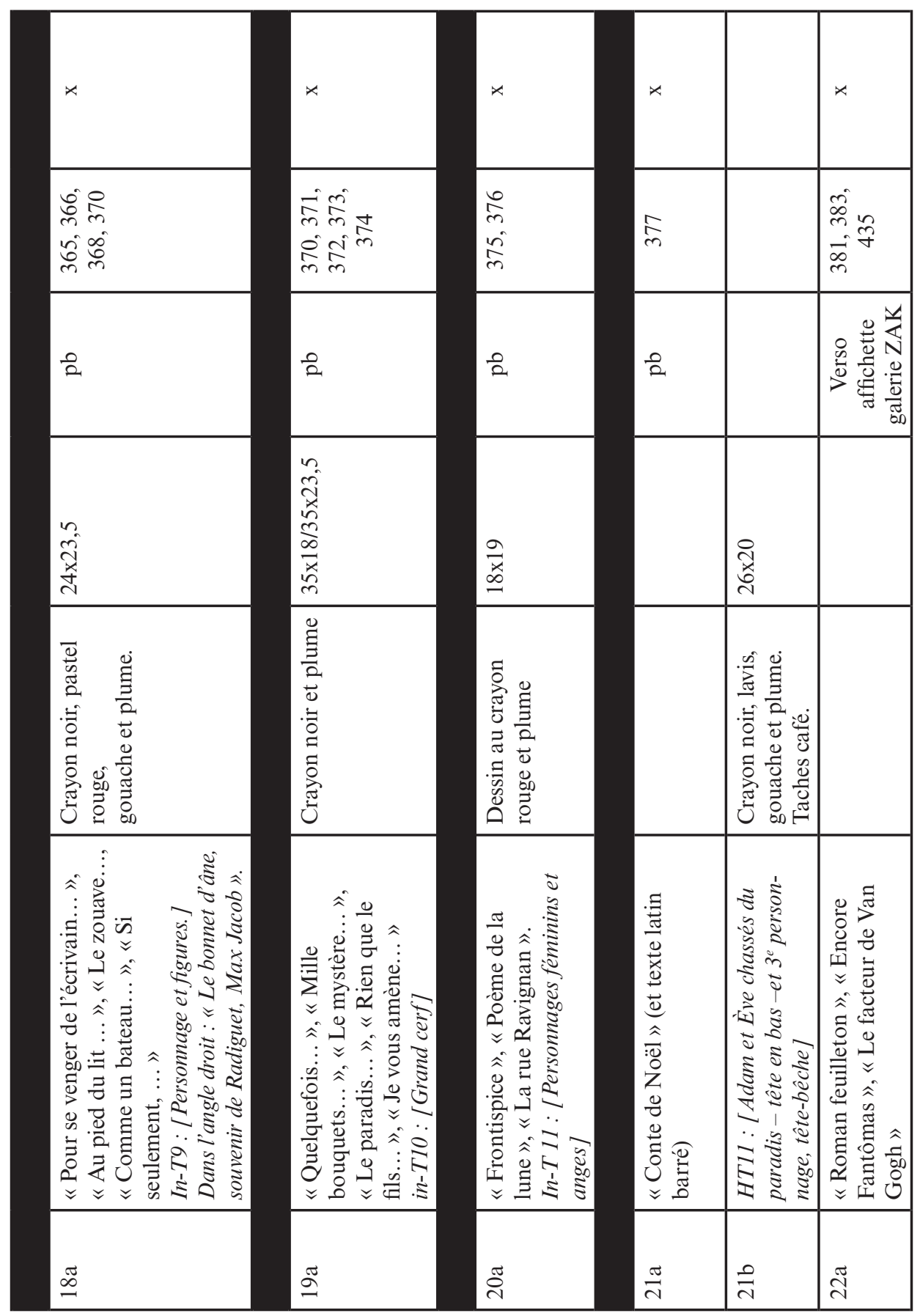




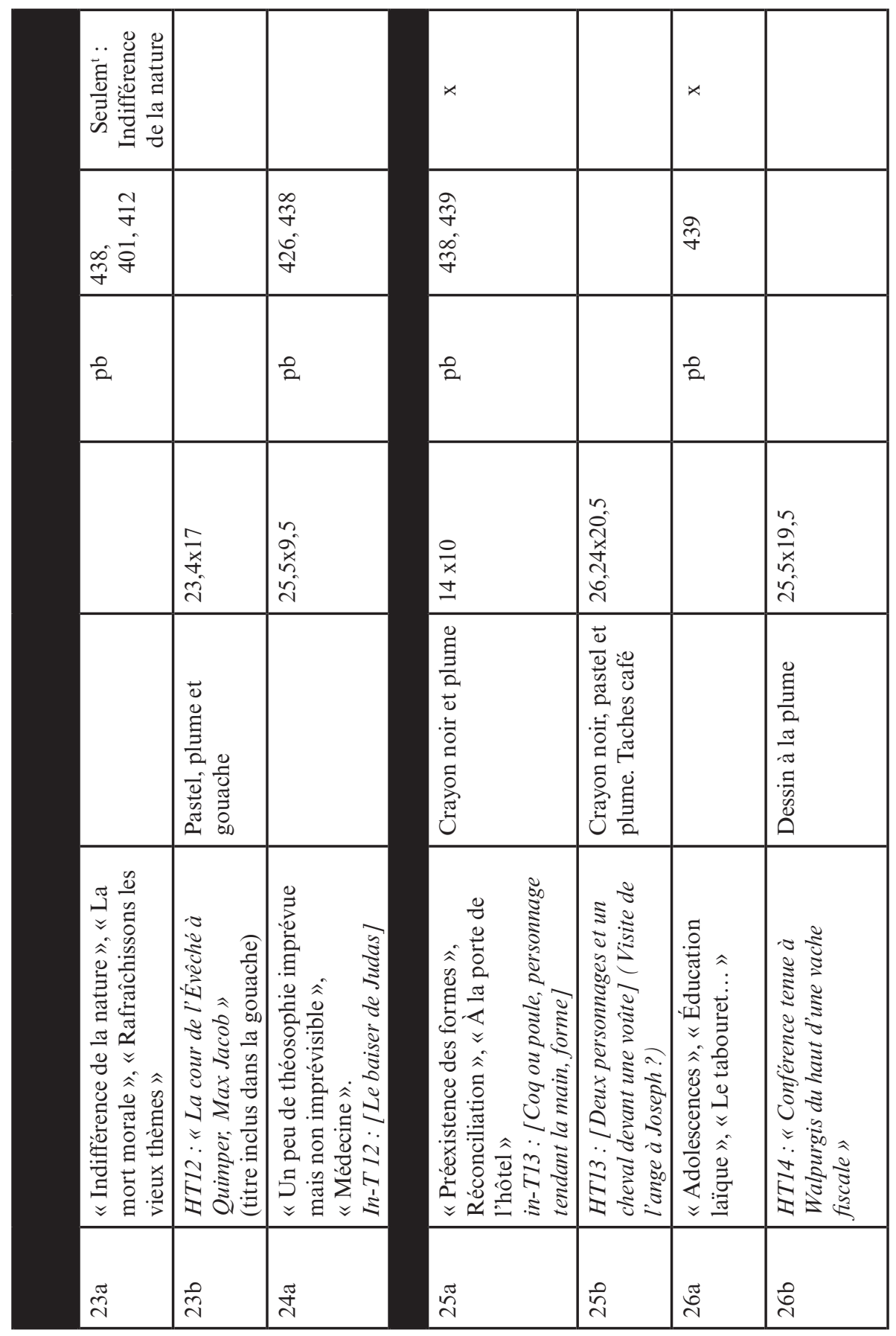

